International Journal of Pure and Applied Mathematics

Volume 91 No. 4 2014, 495-504

ISSN: 1311-8080 (printed version); ISSN: 1314-3395 (on-line version)

url: http://www.ijpam.eu

doi: http://dx.doi.org/10.12732/ijpam.v91i4.6

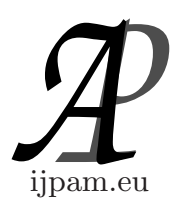

\title{
PRODUCT CORDIAL LABELING OF GRAPHS RELATED TO HELM, CLOSED HELM AND GEAR GRAPH
}

\author{
G.V. Ghodasara ${ }^{1}$, S.M. Vaghasiya ${ }^{2}$ \\ ${ }^{1}$ H.\&H.B. Kotak Institute of Science \\ Rajkot, Gujarat, INDIA \\ ${ }^{2}$ R.K. University \\ Rajkot, Gujarat, INDIA
}

\begin{abstract}
We prove that path union of finite copies of helm, closed helm, gear graph are product cordial graphs. Further we prove that the graph obtained by joining two copies of helm by a path of arbitrary length is product cordial graph. We prove similar results for closed helm and gear graph.
\end{abstract}

AMS Subject Classification: $05 \mathrm{C} 78$

Key Words: product cordial labeling, helm, closed helm, gear graph

\section{Introduction}

We consider simple, finite, undirected graph $G=(V, E)$. In this paper $P_{n}$ denotes path with $n$ vertices. For all other terminology and notations we follow Harary[1]. First we will provide some definitions useful for the present work.

Definition 1. Let $G$ be a graph and $G_{1}, G_{2}, \ldots, G_{n}, n \geq 2$ be $n$ copies of graph $G$. The graph obtained by adding an edge from $G_{i}$ to $G_{i+1}$ (for $i=$ $1,2, \ldots, n-1)$ is called path union of $G$.

Definition 2. If the vertices of the graph are assigned values subject to certain conditions is known as graph labeling.

Detailed survey on graph labeling is given and updated by Gallian[2].

Received: December 4, 2013

(C) 2014 Academic Publications, Ltd.

${ }^{\S}$ Correspondence author url: www.acadpubl.eu 
Definition 3. Let $G=(V, E)$ be a graph. A mapping $f: V(G) \rightarrow\{0,1\}$ is called binary vertex labeling of $G$ and $f(v)$ is called label of the vertex $v$ of $G$ under $f$.

Definition 4. A binary vertex labeling $f: V(G) \rightarrow\{0,1\}$ of graph $G$ with induced edge labeling $f^{*}: E(G) \longrightarrow\{0,1\}$ defined by $f^{*}(u v)=f(u) f(v)$ is called a product cordial labeling if $\left|v_{f}(0)-v_{f}(1)\right| \leq 1$ and $\left|e_{f}(0)-e_{f}(1)\right| \leq$ 1 , where $v_{f}(0), v_{f}(1)$ denote the number of vertices of $G$ having labels 0,1 respectively under $f$ and $e_{f}(0), e_{f}(1)$ denote the number of edges of $G$ having labels 0,1 respectively under $f^{*}$.

A graph $G$ is product cordial if it admits product cordial labeling.

Definition 5. A helm $H_{n}, n \geq 3$ is the graph obtained from the wheel $W_{n}$ by adding a pendant edge at each vertex on the rim of the wheel $W_{n}$.

Definition 6. A closed helm $\mathrm{CH}_{n}$ is the graph obtained by taking a helm $H_{n}$ and adding edges between the pendant vertices.

Definition 7. A gear graph is obtained from the wheel $W_{n}$ by adding a vertex between every pair of adjacent vertices of rim of the wheel $W_{n}$.

The concept of product cordial labeling was introduced by R. Ponraj, M. Sivakumar, M. Sundaram[3]. Vaidya and Barasara[4] proved that friendship graph, cycle with one chord, cycle with twin chord are product cordial graphs. They also proved middle graph of path $P_{n}$ is product cordial graph. Vaidya and Dani[5] proved that the graph obtained by joining apex vertices of two stars is product cordial. They also proved similar results for shell and wheel. In [6], same authors proved that path union of $k$ copies of cycle $C_{n}$, the graph obtained by joining two copies of cycle $C_{n}$ by path $P_{k}$, the path union of $k$ copies of $D_{2}\left(C_{n}\right)$ are product cordial graphs. Vaidya and Vyas[7] proved that the graphs obtained by joining the connected components of respective graphs by a path of arbitrary length is product cordial.

In the present paper we discuss cordial labeling for path union of helm, path union of closed helm and path union of gear graph. Further we prove that the graph obtained by joining two copies of helm by path of arbitrary length is product cordial. Similar results are investigated for closed helm and gear graph.

\section{Main Results}

Theorem 1. The path union of $k$ copies of helm $H_{n}$ admits product cordial labeling. 
Proof. Let $G$ be the path union of $k$ copies $G_{1}, G_{2}, \ldots, G_{k}$ of helm $H_{n}$. Let $\left\{v_{i 0}, v_{i 1}, \ldots, v_{i n}, v_{i 1}^{\prime}, v_{i 2}^{\prime}, \ldots, v_{i n}^{\prime}\right\}$ denote the vertices of $G$. Let $e_{i}=v_{i 0} v_{(i+1) 0}$ be the edge joining $G_{i}$ and $G_{i+1}$. where $v_{i 0}$ is apex vertex, $\left\{v_{i 1}, v_{i 2}, \ldots, v_{i n}\right\}$ are internal vertices and $\left\{v_{i 1}^{\prime}, v_{i 2}^{\prime}, \ldots, v_{i n}^{\prime}\right\}$ are external vertices. We define labeling function $f: V(G) \rightarrow\{0,1\}$ as follows.

Case 1: $k$ is odd.

Subcase 1: $n$ is odd.

$$
\begin{aligned}
& f\left(v_{i j}\right)=1 ; 1 \leq i \leq \frac{k-1}{2}, 0 \leq j \leq n \\
& =0 ; \frac{k+3}{2} \leq i \leq k \leq, 0 \leq j \leq n \\
& f\left(v_{i j}\right)^{\prime}=1 ; 1 \leq i \leq \frac{k-1}{2}, 1 \leq j \leq n \\
& =0 ; \frac{k+3}{2} \leq i \leq k, 1 \leq j \leq n \\
& f\left(v_{\left(\frac{k+1}{2}\right) 1}\right)=0, f\left(v_{\left(\frac{k+1}{2}\right) 1}\right)^{\prime}=0, f\left(v_{\left(\frac{k+1}{2}\right) 2}\right)^{\prime}=0 \\
& f\left(v_{\left(\frac{k+1}{2}\right) j}\right)=1 ; 0 \leq j \leq \frac{n+3}{2} \\
& =0 ; \frac{n+5}{2} \leq j \leq n \\
& \left.f\left(v_{\frac{k+1}{2}}\right) j\right)^{\prime}=1 ; 3 \leq j \leq \frac{n+3}{2} \\
& =0 ; \frac{n+5}{2} \leq j \leq n
\end{aligned}
$$

It can be easily seen that the vertex conditions and edge conditions of product cordial labeling are satisfied in this case, i.e. $\left|v_{f}(1)-v_{f}(0)\right| \leq 1$ and $\mid e_{f}(1)-$ $e_{f}(0) \mid \leq 1$.

Subcase 2: $n$ is even.

$$
\begin{aligned}
& f\left(v_{i j}\right)=1 ; 1 \leq i \leq \frac{k-1}{2}, 0 \leq j \leq n \\
& =0 ; \frac{k+3}{2} \leq i \leq k \leq, 0 \leq j \leq n \\
& f\left(v_{i j}\right)^{\prime}=1 ; 1 \leq i \leq \frac{k-1}{2}, 1 \leq j \leq n \\
& =0 ; \frac{k+3}{2} \leq i \leq k, 1 \leq j \leq n \\
& f\left(v_{\left(\frac{k+1}{2}\right) 1}\right)=0, f\left(v_{\left(\frac{k+1}{2}\right) 1}\right)^{\prime}=0, f\left(v_{\left(\frac{k+1}{2}\right) 2}\right)^{\prime}=0, f\left(v_{\left(\frac{k+1}{2}\right) 3}\right)^{\prime}=0 \\
& f\left(v_{\left(\frac{k+1}{2}\right) j}\right)=1 ; 0 \leq j \leq \frac{n+4}{2}, j \neq 1 \\
& =0 ; \frac{n+6}{2} \leq j \leq n \\
& f\left(v_{\left.\frac{k+1}{2}\right) j}\right)^{\prime}=1 ; 4 \leq j \leq \frac{n+4}{2} \\
& =0 ; \frac{n+6}{2} \leq j \leq n
\end{aligned}
$$

It can be easily seen that the vertex conditions and edge conditions of product cordial labeling are satisfied in this case, i.e. $\left|v_{f}(1)-v_{f}(0)\right| \leq 1$ and $\mid e_{f}(1)-$ $e_{f}(0) \mid \leq 1$.

Case 2: $k$ is even, $\forall n$

$f\left(v_{i j}\right)=1 ; 1 \leq i \leq \frac{k}{2}, 0 \leq j \leq n$ 


$$
\begin{aligned}
& =0 ; \frac{k}{2}+1 \leq i \leq k, 0 \leq j \leq n \\
f\left(v_{i j}\right)^{\prime} & =1 ; 1 \leq i \leq \frac{k}{2}, 1 \leq j \leq n \\
& =0 ; \frac{k}{2}+1 \leq i \leq k, 1 \leq j \leq n
\end{aligned}
$$

It can be easily seen that the vertex conditions and edge conditions of product cordial labeling are satisfied in this case, i.e. $\left|v_{f}(1)-v_{f}(0)\right| \leq 1$ and $\mid e_{f}(1)-$ $e_{f}(0) \mid \leq 1$.

Hence in each case the graph $G$ under consideration satisfies the conditions for product cordial labeling. Hence $G$ is a product cordial graph.

Example 1. The product cordial labeling of path union of 3-copies of helm $H_{8}$ is shown in Figure 1. It is the case related to $k$ is odd, $n$ is even.

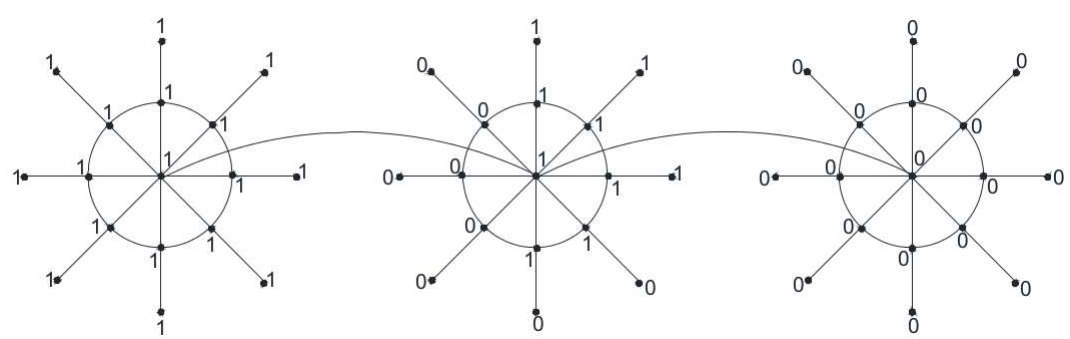

Figure 1: Product cordial labeling of path union of 3 copies of $H_{8}$

Theorem 2. The path union of $k$ copies of closed helm $C H_{n}$ admits product cordial labeling.

Proof. Let $G$ be the path union of $k$ copies $G_{1}, G_{2}, \ldots, G_{k}$ of closed helm $C H_{n}$. Let $\left\{v_{i 0}, v_{i 1}, \ldots, v_{i n}, v_{i 1}^{\prime}, v_{i 2}^{\prime}, \ldots, v_{i n}^{\prime}\right\}$ denote the vertices of $G$. Let $e_{i}=$ $v_{i 0} v_{(i+1) 0}$ be the edge joining $G_{i}$ and $G_{i+1}$ where $v_{i 0}$ is apex vertex, $\left\{v_{i 1}, \ldots, v_{i n}\right\}$ are internal vertices and $\left\{v_{i 1}^{\prime}, v_{i 2}^{\prime}, \ldots, v_{i n}^{\prime}\right\}$ are external vertices. We define labeling function $f: V(G) \rightarrow\{0,1\}$ as follows.

Case 1: $k$ is odd.

$$
\begin{aligned}
& f\left(v_{i j}\right)=1 ; 1 \leq i \leq \frac{k-1}{2}, 0 \leq j \leq n \\
& =0 ; \frac{k+3}{2} \leq i \leq k, 0 \leq j \leq n \\
& f\left(v_{i j}\right)^{\prime}=1 ; 1 \leq i \leq \frac{k-1}{2}, 1 \leq j \leq n \\
& =0 ; \frac{k+3}{2} \leq i \leq k, 1 \leq j \leq n \\
& f\left(v_{\left(\frac{k+1}{2}\right) j}\right)=1 ; 0 \leq j \leq n \\
& f\left(v_{\left(\frac{k+1}{2}\right) j}\right)^{\prime}=0 ; 1 \leq j \leq n .
\end{aligned}
$$


Case 2: $k$ is even.

$$
\begin{aligned}
f\left(v_{i j}\right) & =1 ; 1 \leq i \leq \frac{k}{2}, 0 \leq j \leq n \\
& =0 ; \frac{k}{2}+1 \leq i \leq k, 0 \leq j \leq n \\
f\left(v_{i j}\right)^{\prime} & =1 ; 1 \leq i \leq \frac{k}{2}, 1 \leq j \leq n \\
& =0 ; \frac{k}{2}+1 \leq i \leq k, 1 \leq j \leq n
\end{aligned}
$$

The graph $G$ under consideration satisfies the conditions $\left|v_{f}(1)-v_{f}(0)\right| \leq 1$ and $\left|e_{f}(1)-e_{f}(0)\right| \leq 1$ in each case. Hence the graph $G$ is product cordial graph.

Example 2. The product cordial labeling of the path union of 3-copies of closed helm $\mathrm{CH}_{5}$ is shown in Figure 2. It is the case related to $k$ is odd.

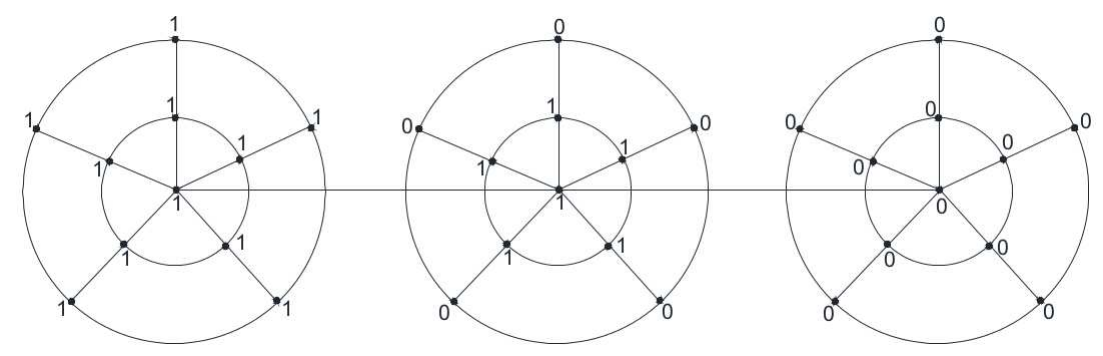

Figure 2: Product cordial labeling of path union of 3 copies of closed helm $\mathrm{CH}_{5}$.

Theorem 3. The path union of $k$ copies of gear graph $G_{n}$ admits product cordial labeling, when $n$ is odd.

Proof. Let $G$ be the path union of $k$ copies $G_{1}, G_{2}, \ldots, G_{k}$ of gear graph $G_{n}$. Let $\left\{v_{i 0}, v_{i 1}, \ldots, v_{i 2 n}\right\}$ denote the vertices of $G$. where $v_{i 0}$ is apex vertex. Let $e_{i}=v_{i 0} v_{(i+1) 0}$ be the edge joining $G_{i}$ and $G_{i+1}$. We define labeling function $f: V(G) \rightarrow\{0,1\}$ as follows.

Case 1: $k$ is odd, $n$ is odd.

$$
\begin{gathered}
f\left(v_{i j}\right)=1 ; 1 \leq i \leq \frac{k-1}{2}, 0 \leq j \leq 2 n \\
=0 ; \frac{k+3}{2} \leq i \leq k, 0 \leq j \leq 2 n \\
\begin{array}{c}
f\left(v_{\left(\frac{k+1}{2}\right) j}\right)=1 ; 0 \leq j \leq 2 n \\
=0 ; n+1 \leq j \leq 2 n .
\end{array}
\end{gathered}
$$

Case 2: $k$ is even, $n$ is odd.

$$
\begin{aligned}
f\left(v_{i j}\right) & =1 ; 1 \leq i \leq \frac{k}{2}, 0 \leq j \leq 2 n \\
& =0 ; \frac{k}{2}+1 \leq i \leq k, 0 \leq j \leq 2 n
\end{aligned}
$$


The graph $G$ under consideration satisfies the condition $\left|v_{f}(1)-v_{f}(0)\right| \leq 1$ and $\left|e_{f}(1)-e_{f}(0)\right| \leq 1$ in each case.

Hence the graph $G$ is product cordial graph.

Example 3. The product cordial labeling of the path union of 3-copies of gear graph $G_{3}$ is shown in Figure 3. It is the case related to $k$ is odd.

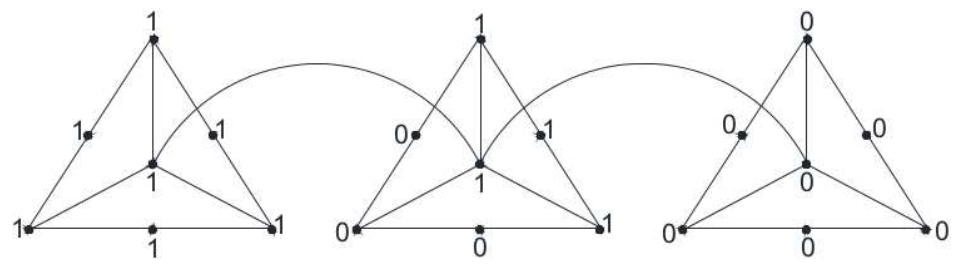

Figure 3: Product cordial labeling of path union of 3 copies of gear graph $G_{3}$.

Theorem 4. The graph obtained by joining two copies of helm $H_{n}$ by a path of arbitrary length admits product cordial labeling.

Proof. Let $G$ be the graph obtained by joining two copies of helm $H_{n}$ by path $P_{k}$ of length $k-1$. Let $\left\{u_{0}, u_{1}, \ldots, u_{n}, u_{1}^{\prime}, u_{2}^{\prime}, \ldots, u_{n}^{\prime}\right\}$ denote the consecutive vertices of first copy of helm $H_{n}$. Here $u_{0}$ is the apex vertex, $\left\{u_{0}, u_{1}, \ldots, u_{n}\right\}$ be the internal vertices and $\left\{u_{0}^{\prime}, u_{1}^{\prime}, \ldots, u_{n}^{\prime}\right\}$ be the external(pendant) vertices. Similarly let $\left\{w_{0}, w_{1}, \ldots, w_{n}, w_{1}^{\prime}, w_{2}^{\prime}, \ldots, w_{n}^{\prime}\right\}$ denote the consecutive vertices of second copy of helm $H_{n}$, where $w_{0}$ is the apex vertex, $\left\{w_{0}, w_{1}, \ldots, w_{n}\right\}$ be the internal vertices and $\left\{w_{0}^{\prime}, w_{1}^{\prime}, \ldots, w_{n}^{\prime}\right\}$ be the external(pendant) vertices. Let $\left\{v_{1}, v_{2}, \ldots, v_{k}\right\}$ denote the vertices of path $P_{k}$ with $u_{0}=v_{1}$ and $v_{k}=w_{0}$. First we label the vertices of first copy of $H_{n}$ by label 1 and label the vertices of second copy of $H_{n}$ by label 0 . At this stage the vertex conditions and the edge conditions of product cordial labeling are satisfied. Now the remaining task is to label the vertices of path $P_{k}$ for which we define labeling function $f: V(G) \rightarrow\{0,1\}$ as follows.

Case 1: $k$ is even. In this case we label the vertices as:

$f\left(v_{i}\right)=1 ; 1 \leq i \leq \frac{k}{2}$

$$
=0 ; \frac{k}{2}+1 \leq i \leq k
$$

It can easily seen that the vertex condition and edge conditions of product cordial labeling are satisfied in this case.

Case 2: $k$ is odd. In this case we label the vertices as: $f\left(v_{i}\right)=1 ; 1 \leq i \leq \frac{k+1}{2}$ 


$$
=0 ; \frac{k+3}{2} \leq i \leq k
$$

It can easily seen that the vertex condition and edge conditions of product cordial labeling are satisfied in this case. Hence the graph $G$ is product cordial graph.

Example 4. Product cordial labeling of the graph obtaining by joining two copies of helm $H_{4}$ by path $P_{5}$ is shown in Figure 4. It is the case related to $k$ is odd.

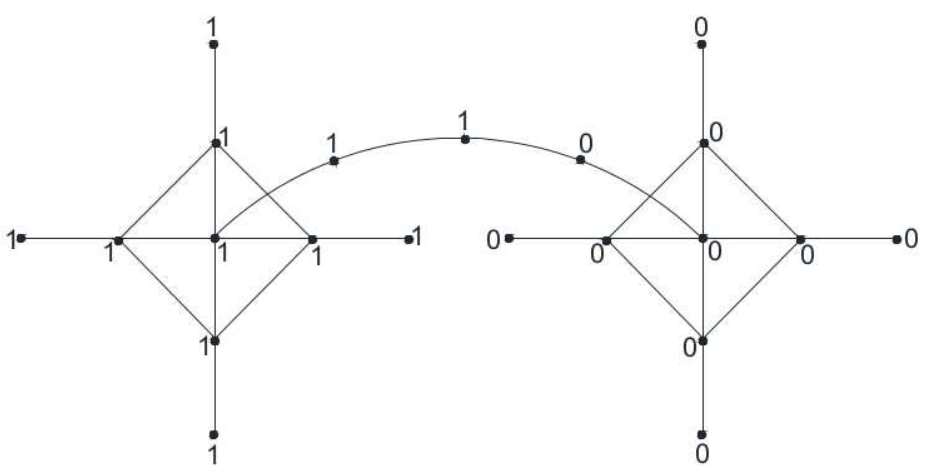

Figure 4: Product cordial labeling of the graph obtained by joining two copies of helm $H_{4}$ by path $P_{5}$.

Theorem 5. The graph obtaining by joining two copies of closed helm $\mathrm{CH}_{n}$ by path of arbitrary length admits product cordial labeling.

Proof. Let $G$ be the graph obtained by joining two copies of closed helm $C H_{n}$ by path $P_{k}$ of $k-1$ length. Let $\left\{u_{0}, u_{1}, \ldots, u_{n}, u_{1}^{\prime}, u_{2}^{\prime}, \ldots\right.$, $\left.u_{n}^{\prime}\right\}$ denote the consecutive vertices of first copy of closed helm $C H_{n}$. Here $u_{0}$ is the apex vertex, $\left\{u_{0}, u_{1}, \ldots, u_{n}\right\}$ be the internal vertices and $\left\{u_{0}^{\prime}, u_{1}^{\prime}, \ldots, u_{n}^{\prime}\right\}$ be the external(pendant) vertices. Similarly let $\left\{w_{0}, w_{1}, \ldots, w_{n}, w_{1}^{\prime}, w_{2}^{\prime}, \ldots, w_{n}^{\prime}\right\}$ denote the consecutive vertices of second copy of closed helm $C H_{n}$, where $w_{0}$ is the apex vertex, $\left\{w_{0}, w_{1}, \ldots, w_{n}\right\}$ be the internal vertices and $\left\{w_{0}^{\prime}, w_{1}^{\prime}, \ldots, w_{n}^{\prime}\right\}$ be the external(pendant) vertices. Let $\left\{v_{1}, v_{2}, \ldots, v_{k}\right\}$ denote the vertices of the path $P_{k}$ with $u_{0}=v_{1}$ and $v_{k}=w_{0}$. First we label the vertices of first copy of $\mathrm{CH}_{n}$ by label 1 and label the vertices of second copy of $C H_{n}$ by label 0 . At this stage the vertex condition and the edge condition of product cordial labeling are satisfied. Now the remaining task is to label the vertices of path $P_{k}$ for which we define labeling function $f: V(G) \rightarrow\{0,1\}$ as follows. 
Case 1: $k$ is even. In this case we label the vertices as: $f\left(v_{i}\right)=1 ; 1 \leq i \leq \frac{k}{2}$

$$
=0 ; \frac{k}{2}+1 \leq i \leq k
$$

It can easily seen that the vertex condition and edge conditions of product cordial labeling are satisfied in this case.

Case 2: $k$ is odd. In this case we label the vertices as:

$f\left(v_{i}\right)=1 ; 1 \leq i \leq \frac{k+1}{2}$

$$
=0 ; \frac{k+3}{2} \leq i \leq k \text {. }
$$

It can easily seen that the vertex condition and edge conditions of product cordial labeling are satisfied in this case. Hence the graph $G$ is product cordial graph.

Example 5. Product cordial labeling of the graph obtained by joining two copies of closed helm $\mathrm{CH}_{5}$ by path $P_{3}$ is shown in Figure 5 . It is the case related to $k$ is odd.

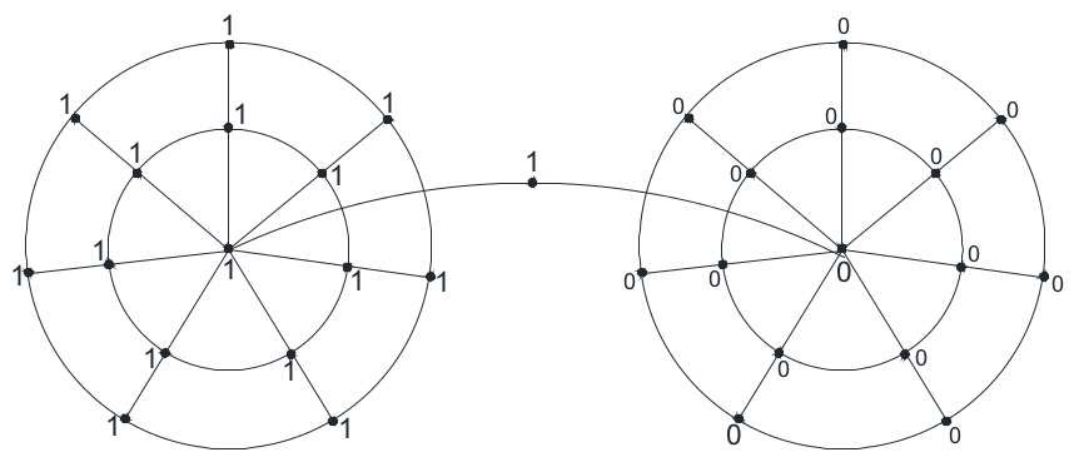

Figure 5: Product cordial labeling of the graph obtained by joining two copies of closed helm $\mathrm{CH}_{7}$ by path $\mathrm{P}_{3}$.

Theorem 6. The graph obtaining by joining two copies of gear $G_{n}$ by path of arbitrary length admits product cordial labeling.

Proof. Let $G$ be the graph obtained by joining two copies of gear graph $G_{n}$ by path $P_{k}$ of $k-1$ length. Let $\left\{u_{0}, u_{1}, \ldots, u_{2 n}\right\}$ denote the consecutive vertices of first copy of gear graph $G_{n}$ and let $\left\{w_{0}, w_{1}, \ldots, w_{2 n}\right\}$ denote the consecutive vertices of second copy of gear graph $G_{n}$. Let $\left\{v_{1}, v_{2}, \ldots, v_{k}\right\}$ denote the vertices of the path $P_{k}$ with $u_{0}=v_{1}$ and $v_{k}=w_{0}$. First we label the vertices of first copy of $G_{n}$ by label 1 and label the vertices of second copy of $G_{n}$ by label 0 . 
At this stage the vertex conditions and the edge conditions of product cordial labeling are satisfied. Now the remaining task is to label the vertices of path $P_{k}$ for which we define labeling function $f: V(G) \rightarrow\{0,1\}$ as follows.

Case 1: $k$ is even

In this case we label the vertices as:

$f\left(v_{i}\right)=1 ; 1 \leq i \leq \frac{k}{2}$

$=0 ; \frac{k}{2}+1 \leq i \leq k$

It can easily seen that the vertex condition and edge condition of product cordial labeling are satisfied in this case.

Case 2: $k$ is odd

In this case we label the vertices as:

$f\left(v_{i}\right)=1 ; 1 \leq i \leq \frac{k+1}{2}$

$=0 ; \frac{k+3}{2} \leq i \leq k$

It can easily seen that the vertex condition and edge condition of product cordial labeling are satisfied in this case.

Hence in each case the graph $G$ under consideration satisfies the conditions of product cordial labeling i.e. $\left|v_{f}(1)-v_{f}(0)\right| \leq 1$ and $\left|e_{f}(1)-e_{f}(0)\right| \leq 1$.

Hence $G$ is product cordial graph.

Example 6. The product cordial labeling of two copies of gear $G_{6}$ joined by path $P_{6}$ is shown in Figure 6 . It is the case related to $k$ is even.

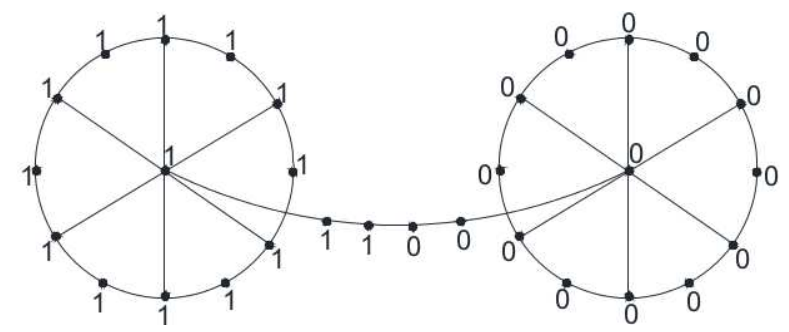

Figure 6: Product cordial labeling of the graph obtaining by joining two copies of gear $G_{6}$ by path $P_{6}$.

\section{Conclusion}

In this paper we investigated six new product cordial graphs. The results proved in this paper are novel. Illustrations are provided at the end of each theorem for better understanding of the labeling pattern defined in each theorem. 


\section{References}

[1] F. Harary, Graph theory, Addision-wesley, Reading, MA (1969).

[2] J. A. Gallian, A dynamic survey of graph labeling, The Electronics Journal of Combinatorics, 19 (2012), $\sharp D S 61-260$.

[3] R. Ponraj, M. Sundaram, M. Sivakumar, $k$-product cordial labeling of graphs, International Journal of contemp. mathematical sciences, 7, No.15(2012), $733-742$.

[4] S. K. Vaidya, C. M. Barasara, Product Cordial Labeling for some New Graphs, Journal of mathematics Research, 3, No.2(2011), 206 - 211, doi:10.5539/jmr.V3n2p206.

[5] S. K. Vaidya, N. A. Dani, Some new product cordial graphs, Journal of Applied computer science and Mathematics, 4, No.8(2010), 62 - 65.

[6] S. K. Vaidya, K. K. Kanani, Some cycle related product cordial graphs, International Journal of Algorithms, computing and Mathematics, $\mathbf{3}$, No.1(2010), $109-116$.

[7] S. K. Vaidya, N. B. Vyas, Product Cordial Labeling in the context of Tensor Product of Graphs, Journal of mathematics Research, 3, No.3(2011), $83-88$, doi:10.5539/jmr.V3n3p83. 